\title{
Respiratory tract infections: uncommon pathogens and misleading presentations
}

\author{
Simon E Brill, Wei Shen Lim and Jeremy S Brown
}

Respiratory infections commonly present to many different clinical specialties, posing diagnostic and therapeutic challenges for a range of physicians. Besides community and hospitalacquired pneumonia there are a variety of more unusual causes of respiratory infections associated with their own particular management problems. This one day conference, organised by Dr Jeremy Brown (University College London) and Dr Wei Shen Lim (University of Nottingham) on behalf of the Royal College of Physicians (RCP) and the British Thoracic Society, was held at the RCP and explored the clinical presentations of these less common respiratory infections.

\section{Uncommon pathogens}

Dr Mark Woodhead discussed the diagnosis and management of pneumonia due to Staphylococcus aureus. This is a topical subject, with anxiety surrounding superinfection with $S$. aureus pneumonia during the recent influenza outbreaks and increasing isolation of strains producing the Panton-Valentin Leucocidin (PVL) toxin. S. aureus pneumonia is classically described as complicating influenza and during the $2009 \mathrm{H} 1 \mathrm{~N} 1$ pandemic around $30 \%$ of patients who died or required intensive care had evidence of a bacterial pneumonia. A post mortem study $^{1}$ from the USA found that staphylococcal pneumonia accounted for one third of bacterial infections identified in fatal cases of pandemic H1N1. PVL toxin can be produced by both community- or hospital-acquired methicillin sensitive or resistant $S$. aureus strains and is associated with a high mortality. Infection with PVL producing S. aureus should be suspected in necrotising cutaneous or lung infections, especially if the patient has close communal contacts associated with skin abrasions (eg through sports teams or the armed forces). Fortunately these infections remain rare within the $\mathrm{UK}$, but early recognition is important as specific antibiotic therapy may be required (eg with clindamycin, rifampicin and/or linezolid). Dr Woodhead reiterated old data ${ }^{2}$ suggesting that the most sensitive test for $S$. aureus pneumonia is a sputum Gram stain - although now rarely requested, clusters of Gram positive bacteria in the

Simon E Brill, specialist registrar in respiratory medicine, Barnet General Hospital; Wei Shen Lim, consultant in respiratory medicine, Nottingham University Hospitals NHS Trust; Jeremy S Brown, reader in respiratory infection, Centre for Respiratory Research, University College London

This conference took place at the Royal College of Physicians (RCP) on 15 March 2011 and was organised by the British Thoracic Society and the RCP. sputum are present in the majority of patients with $S$. aureus pneumonia and this provides a rapid method of confirming the diagnosis.

Advances in the diagnosis and treatment of Aspergillus infection were reviewed by Professor David Denning. Sputum Aspergillus culture has a relatively low sensitivity (about 50\%) for patients with chronic or acute invasive aspergillosis, but data from Professor Denning's laboratory suggest that the yield can be dramatically improved by plating a much higher volume of the sample ( $10 \mathrm{ul}$ instead of $1 \mathrm{ul}$ ) than that recommended by national guidance. He also presented data (in press) showing that polymerase chain reaction for Aspergillus DNA in respiratory samples can be significantly more sensitive than culture. For treatment of invasive aspergillosis, voriconazole has been shown to have a direct survival benefit (up to $15 \%)^{3}$ compared to amphotericin B, and this should remain the first line therapy unless contraindicated.

Professor Rob Miller reminded us of the wide spectrum of atypical radiological presentations seen with Pneumocystis jirovecii pneumonia, which is increasingly seen in non-HIV patients receiving immunosuppression such as prolonged corticosteroid therapy. Treatment options were discussed, with an emphasis placed on using systemic corticosteroids in severe disease to dampen the inflammatory response to Pneumocystis infection to improve the associated hypoxia and reduce mortality.

\section{Misleading presentations}

In the first of the interactive sessions, Professor John Macfarlane considered non-infective causes of consolidation - the 'pneumonia mimics'. The conditions discussed included pulmonary infarction, eosinophilic pneumonias (acute and chronic), pulmonary vasculitides, organising pneumonia, broncho-alveolar cell carcinoma, and alveolar proteinosis. With the exception of pulmonary embolism, these conditions are rare. To illustrate this point the audience ( $75 \%$ of whom were consultants, $63 \%$ in respiratory medicine) were surveyed; over half had not seen a case of eosinophilic pneumonia in the last five years, and most had never seen a case of alveolar proteinosis. However, noninfectious causes remain important differential diagnoses to consider when faced with a case of non-resolving consolidation.

In the second interactive session, Dr Jeremy Brown examined some of the causes of subacute pneumonia. This was illustrated with some cases and images from his own experience including chronic Gram negative pneumonia, actinomycosis, Nocardia, non-tuberculous mycobacteria, and aspergillosis. The 
emphasis was on the importance of seeking a conclusive diagnosis, including early use of bronchoscopy or radiologically guided biopsy for histology which is often diagnostic and also generally excludes lung cancer, one of the main differential diagnoses for these infections.

\section{Are antibiotics indicated in patients admitted with exacerbations of chronic obstructive pulmonary disease?}

There followed a lively debate about the use of antibiotics in patients hospitalised with chronic obstructive pulmonary disease (COPD) exacerbations. Prior to the debate, $73 \%$ of the audience said they would routinely treat with antibiotics - an uphill struggle for Dr Charlotte Bolton to overcome from the start.

Both speakers identified a paucity of data on the subject, with the last Cochrane review (2006) only able to identify three suitable studies. The discussion therefore centred on recently published data, in particular a randomised controlled trial by Daniels and colleagues. ${ }^{4}$ This was a placebo-controlled trial of doxycycline in addition to systemic steroids in a total of 265 exacerbations. In this study, while there was an improvement in the clinical cure at day 10 , this was not sustained at day 30 (Fig 1).

In the discussion, Professor Wisia Wedzicha highlighted the success at 10 days, while arguing that the primary outcome of clinical cure at 30 days is highly heterogeneous and likely to capture clustering or second exacerbations. She also presented data from her group 5 and a large retrospective US cohort $(84,621$ patients), ${ }^{6}$ both suggesting a better outcome with early antibiotic therapy. Dr Bolton focused on the paucity of recent data and the fact that the Daniels et al study was negative when judged by the primary outcome measure. She also highlighted an increased Clostridium difficile incidence in the US cohort. There was some common ground; both agreed that many minor exacerbations are likely to be self-limiting and that clinical or microbiological evidence of bacterial colonisation should be

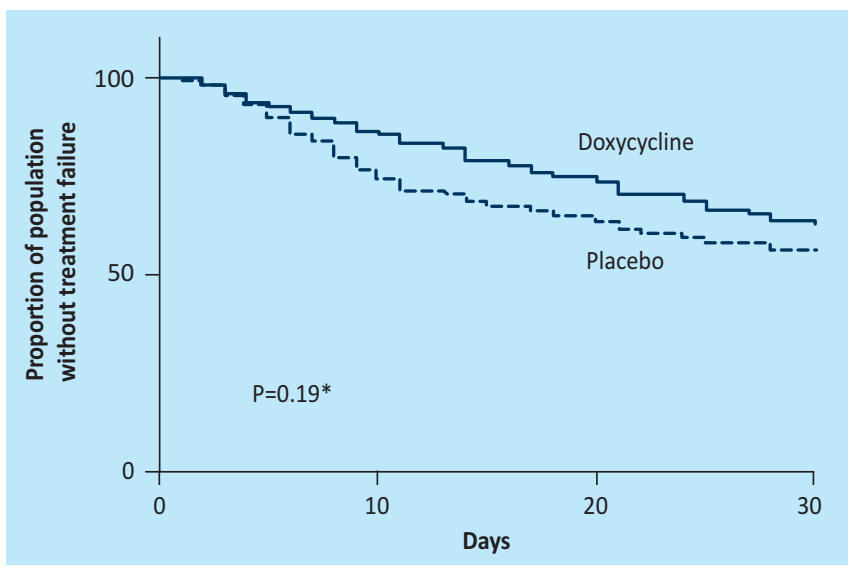

Fig 1. Kaplan-Meier curves showing effect of the intervention (doxycycline) on time to treatment failure in the intention-to-treat population. Reproduced with permission from the American Thoracic Society. ${ }^{4}$ Copyright (C) American Thoracic Society. sought before using antibiotics. Conversely, there was clear agreement that patients with a C-reactive protein (CRP) $>50$ or evidence of bacterial infection should receive antibiotics (Fig 2). Looking to the future, the use of other biomarkers including procalcitonin may help identify patients who would benefit from antibiotics in addition to steroids during exacerbations.

In the subsequent audience poll, 75\% of participants suggested that they would still treat with antibiotics, broadly similar to the pre-debate proportion.

\section{Updates}

Dr Nick Maskell delivered an update on the treatment of pleural infection, a major health problem with a one-year mortality higher than that of myocardial infarction and a combined incidence of 65,000 cases/year in the UK and USA. The highlight was a discussion of the recent MIST-2 trial (in press), a multicentre UK randomised controlled trial of treatment with intrapleural DNAse and tPA in confirmed pleural infection. Patients in the dual treatment arm had a significant improvement in resolution of pleural shadowing. The trial was not powered to identify changes in mortality or surgical intervention rates, but the data warrant a much larger trial.

Dr Wei Shen Lim, who was chair of the joint Pandemic Influenza Clinical Management Guidelines Committee, was ideally placed to deliver an update on what we have learned from the 2009 influenza pandemic. In a study of patients hospitalised in the UK due to the $2009 \mathrm{H} 1 \mathrm{~N} 1$ influenza, 51\% were female and around $15 \%$ were admitted to critical care or died. ${ }^{7}$ Interestingly, the age distribution of hospitalised cases in the UK was heavily weighted in favour of those under 65 years old. In 1918, H1N1 influenza A caused the 'Spanish' influenza pandemic, and then remained in circulation globally until 1957 when it was replaced by the H2N2 strain that caused the 'Asian' flu pandemic. Therefore, older members of the population who had been exposed to previous $\mathrm{H} 1 \mathrm{~N} 1$ strains $^{8}$ were partially immune to influenza A H1N1 2009. Worryingly, oseltamivir resistance was observed, albeit uncommonly, in H1N1 2009 influenza A infection, including person-to-person transmission of the resistant strain. Although the first wave of the $2009 \mathrm{H} 1 \mathrm{~N} 1$ pandemic occurred in spring, it caused a similar number of deaths as the winter 2010-11 seasonal outbreak in the $\mathrm{UK}^{9}$ (although considerably fewer than the 1999 seasonal flu epidemic). In admitted patients, asthma was the most common co-morbidity, and physician-recorded obesity or pulmonary conditions other than asthma or chronic obstructive pulmonary disease were associated with a poorer outcome. If influenza A H1N1 2009 behaves in a similar way to previous pandemic viruses, this strain will become established as the main circulating influenza A strain; how this might affect the usual winter mortality spike due to endemic influenza in the elderly remains to be seen.

The last session was an interactive update on ventilatoracquired pneumonia (VAP) from Professor John Simpson. The diagnosis of VAP is notoriously difficult and Professor Simpson presented data from his own group suggesting that 


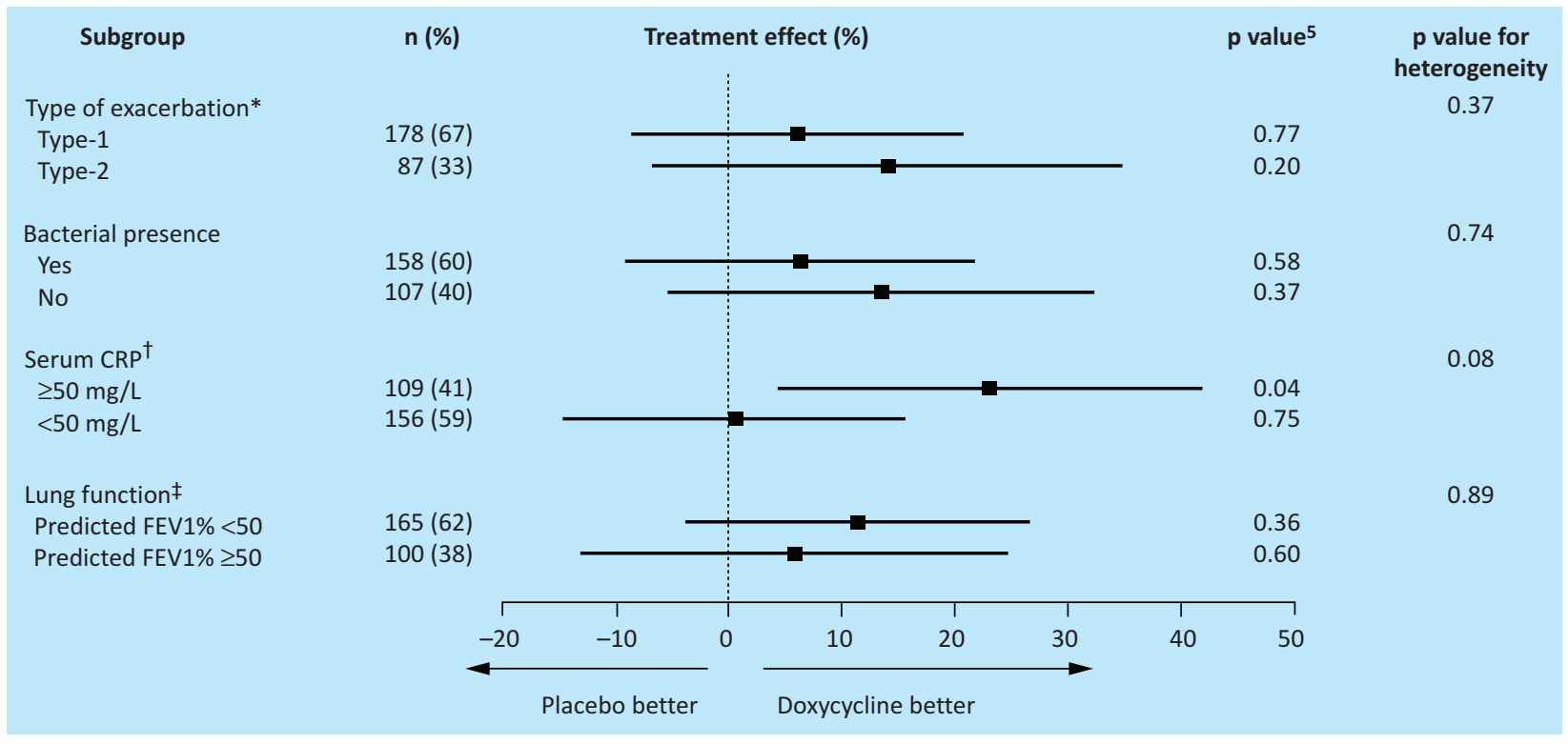

Fig 2. Subgroup analysis on day 30. CRP = C-reactive protein; FEV1 = forced expiratory volume in 1 second. Reproduced with permission from the American Thoracic Society. ${ }^{4}$ Copyright (c) American Thoracic Society.

\section{Conference programme}

Chair: Dr Wei Shen Lim, Nottingham University Hospitals NHS Trust Uncommon pathogens

When to suspect staphylococcal (including PVL) pneumonia and initial management

Dr Mark Woodhead, Manchester Royal Infirmary

When to suspect pneumocystis pneumonia and initial management Professor Rob Miller, University College London

When to suspect aspergillus lung infection and initial management Professor David Denning, University Hospital of South Manchester

\section{Misleading presentations}

Consolidation, but not infection?

Eosinophilic pneumonias, organising pneumonias and other mimics Professor John Macfarlane, Nottingham University

Subacute cavitating pneumonias - TB, environmental Mycobacteria, or something else?

Dr Jeremy Brown, University College London Hospital

Chair: Dr Jeremy Brown

Debate: Antibiotics are indicated in patients admitted with exacerbations of COPD

For: Professor Wisia Wedzicha, UCL Medical School

Against: Dr Charlotte Bolton, Nottingham Respiratory Biomedical

Research Unit

Updates

Parapneumonic effusions - recent advances in knowledge

Dr Nick Maskell, University of Bristol

What have we learnt from the 2009 A/H1N1 pandemic? Dr Wei Shen Lim

Hospital-acquired pneumonia: from ward to ICU/current challenges Professor John Simpson, Newcastle University bronchoalveolar lavage levels of specific cytokines may have useful negative and positive predictive values. In addition, he discussed prevention of VAP using 'bundles' of simple targeted interventions.

The talks from nationally and internationally recognised experts provoked discussion between the audience and speakers, considerably enhancing the educational benefit of the conference for both groups. Overall the conference demonstrated the wide range of presentations of different respiratory tract infections and the high degree of interest in the subject among clinicians. Lung infections are important medical problems that remain highly relevant for healthcare in the 21st century.

\section{References}

1 Centers for Disease Control and Prevention (CDC). Bacterial coinfections in lung tissue specimens from fatal cases of 2009 pandemic influenza A (H1N1) - United States, May-August 2009. Morb Mortal Wkly Rep 2009;58:1071-4.

2 Woodhead MA, Radvan J, Macfarlane JT. Adult community-acquired staphylococcal pneumonia in the antibiotic era: a review of 61 cases. QJM 1987;64:783-90.

3 Herbrecht R, Denning DW, Patterson TF et al. Voriconazole versus Amphotericin B for primary therapy of invasive aspergillosis. $N$ Engl $J$ Med 2002;347:408-15.

4 Daniels JMA, Snijders D, de Graaff CS et al. Antibiotics in addition to systemic corticosteroids for acute exacerbations of chronic obstructive pulmonary disease. Am J Respir Crit Care Med 2010;181:150-7.

5 Wilkinson TMA, Donaldson GC, Hurst JR, Seemungal TAR, Wedzicha JA. Early therapy improves outcomes of exacerbations of chronic obstructive pulmonary disease. Am J Respir Crit Care Med 2004;169:1298-303.

6 Rothberg MB, Pekow PS, Lahti M et al. Antibiotic therapy and treatment failure in patients hospitalized for acute exacerbations of chronic obstructive pulmonary disease. JAMA 2010;303:2035-42. 
7 Rahman NM, Maskell NA, West A et al. Intrapleural use of tissue plasminogen activator and DNase in pleural infection. $N$ Engl J Med 2011;365:518-26.

8 Nguyen-Van-Tam JS, Openshaw PJ, Hashim A et al; Influenza Clinical Information Network (FLU-CIN). Risk factors for hospitalisation and poor outcome with pandemic A/H1N1 influenza: United Kingdom first wave (May-September 2009). Thorax 2010;65:645-51.

9 Hancock K, Veguilla V, Lu X et al. Cross-reactive antibody responses to the 2009 pandemic H1N1 influenza virus. N Engl J Med 2009;361: 1945-52.

10 Health Protection Agency data. www.hpa.org.uk/Topics/ InfectiousDiseases/InfectionsAZ/PandemicInfluenza/

Address for correspondence: Dr SE Brill,

7b Ospringe Road, London NW5 2JD.

Email: simon.brill@nhs.net

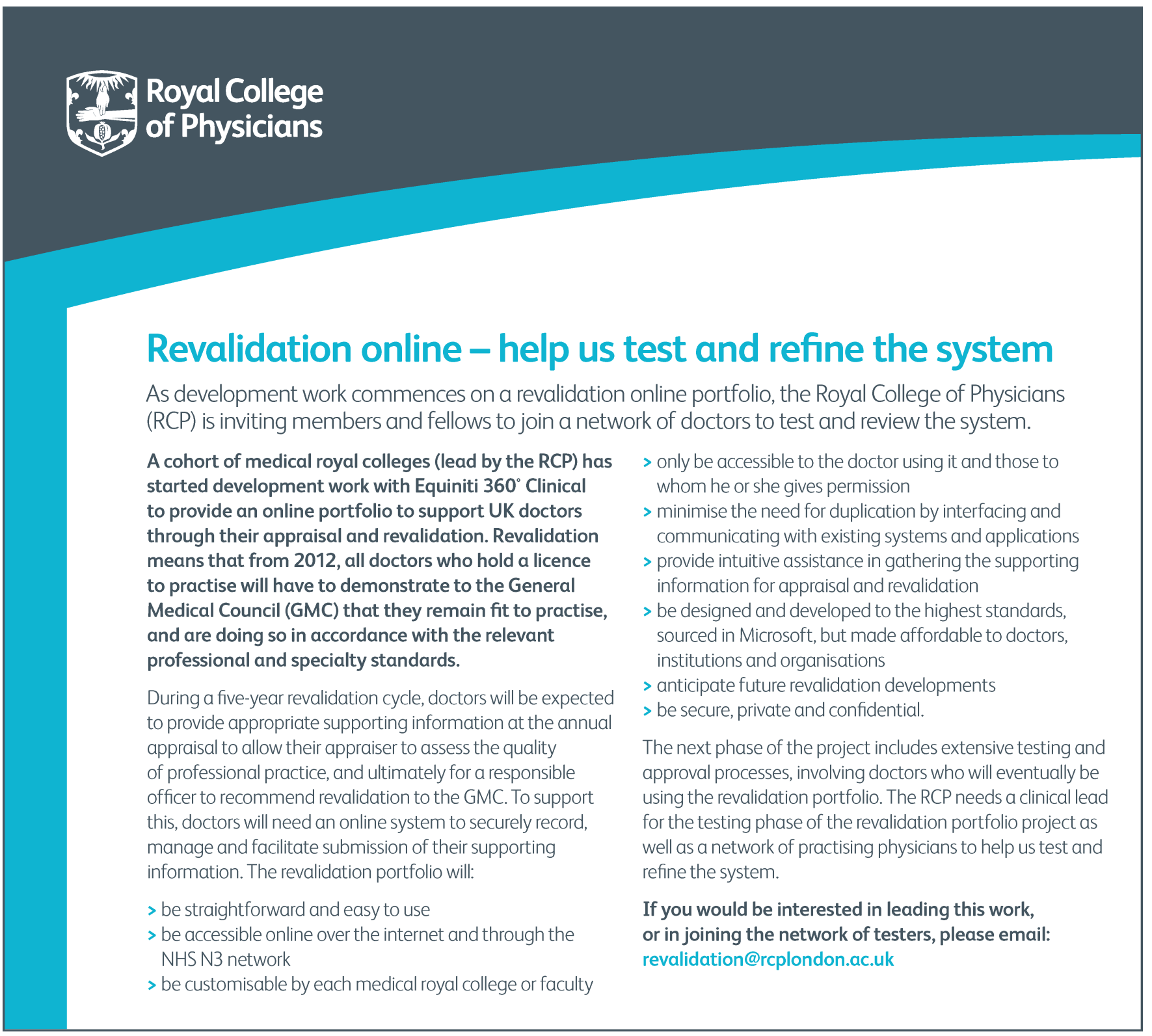

